\title{
Point Defects in Pb-, Bi-, and In-Doped CdZnTe Detectors: Deep-Level Transient Spectroscopy (DLTS) Measurements
}

\author{
R. GUL, ${ }^{1,5}$ K. KEETER, ${ }^{2}$ R. RODRIGUEZ, ${ }^{2}$ A.E. BOLOTNIKOV,${ }^{1}$ \\ A. HOSSAIN,${ }^{1}$ G.S. CAMARDA, ${ }^{1}$ K.H. KIM,${ }^{1}$ G. YANG,${ }^{1}{ }^{\text {Y. CUI, }}{ }^{1}$ \\ V. CARCELEN, ${ }^{3}$ J. FRANC, ${ }^{4}$ Z. LI, ${ }^{1}$ and R.B. JAMES ${ }^{1}$ \\ 1.-Brookhaven National Laboratory, Upton, NY 11973, USA. 2.-Idaho State University, \\ Pocatello, ID 83209, USA. 3.-Universidad Autónoma de Madrid, Madrid, Spain. 4.-Charles \\ University, Prague, Czech Republic. 5.—e-mail: gul@bnl.gov
}

We studied, by current deep-level transient spectroscopy (I-DLTS), point defects induced in CdZnTe detectors by three dopants: $\mathrm{Pb}, \mathrm{Bi}$, and In. $\mathrm{Pb}$-doped CdZnTe detectors have a new acceptor trap at around $0.48 \mathrm{eV}$. The absence of a $\mathrm{V}_{\mathrm{Cd}}$ trap suggests that all Cd vacancies are compensated by $\mathrm{Pb}$ interstitials after they form a deep-acceptor complex $\left[\left[\mathrm{Pb}_{\mathrm{Cd}}\right]^{+}-\mathrm{V}_{\mathrm{Cd}}^{2-}\right]^{-}$. Bi-doped CdZnTe detectors had two distinct traps: a shallow trap at around $36 \mathrm{meV}$ and a deep donor trap at around $0.82 \mathrm{eV}$. In detectors doped with In, we noted three well-known traps: two acceptor levels at around $0.18 \mathrm{eV}$ (A-centers) and $0.31 \mathrm{eV}\left(\mathrm{V}_{\mathrm{Cd}}\right)$, and a deep trap at around $1.1 \mathrm{eV}$.

Key words: CdZnTe detectors, dopant, point defects, DLTS, indium, bismuth, lead, capture cross-section

\section{INTRODUCTION}

Growth of CdZnTe (CZT) ingots from the melt by the Bridgman method yields semi-insulating crystals that hold promise as high-efficiency, highperformance radiation detectors. ${ }^{1-3}$ However, this growth mode entails important drawbacks; viz., the high $\mathrm{Cd}$ partial pressure generates undesirable point defects in the form of $\mathrm{Cd}$ vacancies $\left(\mathrm{V}_{\mathrm{Cd}}\right)$. In addition to these native defects, intentionally added dopants and impurities also introduce some point or complex defects. All of these act as traps for charge carriers within the bandgap by immobilizing a hole or electron. These traps ultimately act as recombination centers, causing annihilation of electrons and holes. Trapping and recombination contribute to the loss of charge carriers and, hence, reduce the average lifetime of the charge carriers. Deep traps close to the midband mainly are responsible for charge loss due to their relatively large cross-section and long detrapping time and, hence, for the degradation of the signal for each event. To ensure high

(Received May 12, 2011; accepted October 17, 2011;

published online November 9, 2011) detector performance and efficiency, the origin of these defects must be identified, so that their density and hence effect can be minimized.

Different dopants are added to CdZnTe crystals during their growth to ensure high resistivity; among these, the most well known are $\mathrm{Pb}, \mathrm{Bi}, \mathrm{In}, \mathrm{Cl}$, $\mathrm{V}, \mathrm{Ge}$, and Sn. In this paper, we report the defects observed in such crystals, based on the type of dopant added. Our main goal is to identify and understand the DLTS signatures of $\mathrm{Pb}-, \mathrm{Bi}-$, and In-related carrier traps and their capture crosssections in $\mathrm{CZT}: \mathrm{Pb}, \mathrm{CZT}: \mathrm{Bi}$, and CZT:In detectors. To study these deep levels, we employed BNL's IDLTS system, ${ }^{4}$ which was designed especially to explore high-resistivity materials, such as CdZnTe, CdMnTe, CdMgTe, TlBr, and heavily irradiated $\mathrm{Si}$ detectors. Li gives further details relating to its theoretical principles. ${ }^{5}$

\section{EXPERIMENTAL PROCEDURES}

We used three types of detectors obtained from different manufacturers: one $\mathrm{Pb}$-doped $\mathrm{CdZnTe}$ detector (vertical Bridgman method), ${ }^{6}$ two Bi-doped CdZnTe detectors (vertical Bridgman method with two different dopant concentrations), ${ }^{7}$ and an 
Table I. Specifications, dopant levels, and resistivities of the four detectors

\begin{tabular}{|c|c|c|c|}
\hline Detector & $\begin{array}{l}\text { Dimensions } \\
\quad\left(\mathrm{mm}^{3}\right)\end{array}$ & $\begin{array}{l}\text { Dopant Level } \\
\quad\left(\text { at. } \mathrm{cm}^{-3}\right)\end{array}$ & $\begin{array}{c}\text { Resistivity } \\
\quad(\Omega \mathrm{cm})\end{array}$ \\
\hline $\mathrm{CZT}: \mathrm{Pb}$ & $5 \times 5 \times 2$ & $1 \times 10^{17}$ & $\sim 10^{10}$ \\
\hline CZT:Bi-B & $11 \times 7 \times 3$ & $1 \times 10^{19}$ & $\sim 10^{8}$ \\
\hline CZT:Bi-Bro & $7 \times 5 \times 2$ & $1 \times 10^{17}$ & $\sim 10^{9}$ \\
\hline CZT:In & $5 \times 5 \times 2$ & - & $\sim 10^{10}$ \\
\hline
\end{tabular}
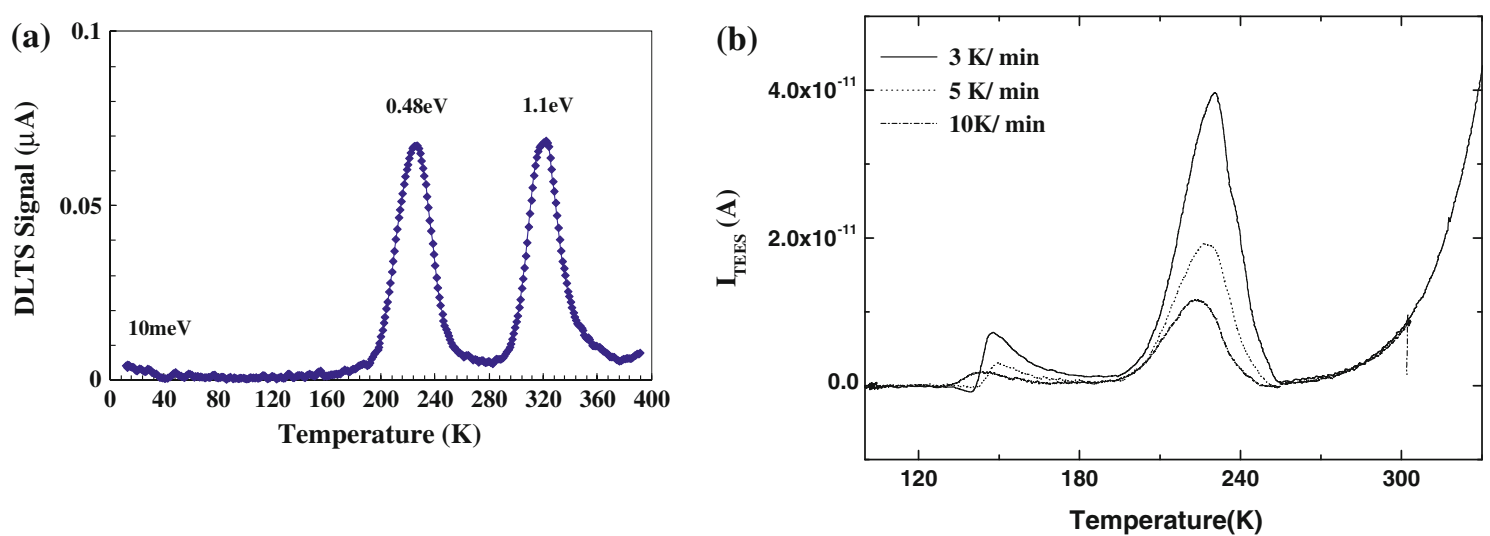

Fig. 1. CZT:Pb detector: (a) DLTS spectrum with defects labeled for $\lambda=822 \mathrm{~nm}$, and (b) TEES measurements with a deep acceptor trap around $230 \mathrm{~K}$.

In-doped CdZnTe detector (Bridgman method). ${ }^{2}$ The $\mathrm{Zn}$ concentration in these detectors was $10 \%$. Table I lists their dimensions, dopant concentrations, and bulk resistivities.

We characterized the point defects in these detectors principally with I-DLTS. To understand the nature of the traps and Te-rich phases in the crystals, some supporting data were obtained from thermal emission electron spectroscopy (TEES) ${ }^{8}$ and infrared (IR) transmission spectroscopy. ${ }^{9} \mathrm{I}-\mathrm{V}$ measurements were carried out to understand the electrical resistivity of the material.

\section{I-DLTS Measurements}

Acquiring I-DLTS measurements involves several steps. In the first step, the sample is set on the cold finger at a particular temperature, and stabilized at that temperature by a temperature controller. Defect filling and emission (trapping and detrapping) are done at each selected temperature. Defect levels are first filled with electrons (or holes) by illuminating the sample with the bias on the sample off, using a laser wavelength with energy less than that of the material's energy bandgap. In the emission process, the laser is shut off, and a bias is applied across the sample. Detrapping of carriers from defect levels generates a transient current. After amplification, the transient current recorded by the oscilloscope is interpreted using a personal computer (PC) with the help of a LabView program. The data are analyzed for the point defects using
IDL and Excel software. A total run taking about $8 \mathrm{~h}$ typically goes from $10 \mathrm{~K}$ to $400 \mathrm{~K}$ with about 390 data points (or transients).

\section{RESULTS AND DISCUSSION}

The DLTS results for the energy traps, the probable origin of the traps, the corresponding capture cross-sections for the carriers $(\sigma)$, and some properties for the three types of CZT detector are described next in detail.

\section{Point Defects}

\section{$C Z T: P b$}

Figure 1 shows the DLTS and TEES plots with energy levels for the CZT:Pb detector. The DLTS plot reveals three energy traps: a shallow trap around $10 \mathrm{meV}$ and two deep traps around $0.48 \mathrm{eV}$ and $1.1 \mathrm{eV}$. According to Savitskya et al., ${ }^{10}$ a shallow trap with $\sim 13 \mathrm{meV}$ binding energy is usually attributed to a metallic impurity, such as $\mathrm{Cu}$ existing in the form of a shallow acceptor $\left[\mathrm{Cu}_{\mathrm{Cd}}\right]^{-}$, or an acceptor complex $\left[\mathrm{V}_{\mathrm{Cd}^{-}} \mathrm{Cu}\right]^{-}$. Looking at the low energy $(10 \mathrm{meV})$ of this trap, we attribute it to metallic impurities, most probably in atomic form, rather than relating it to a complex trap.

A trap around $0.31 \mathrm{eV}$, related to the $\mathrm{V}_{\mathrm{Cd}}$, was absent, pointing to possible compensation of the $\mathrm{V}_{\mathrm{Cd}}$ by substitutional $\mathrm{Pb}$. The $0.48-\mathrm{eV}$ trap was a distinctive energy trap in $\mathrm{Pb}$-doped detectors. There are two possibilities for this: first, there is the 

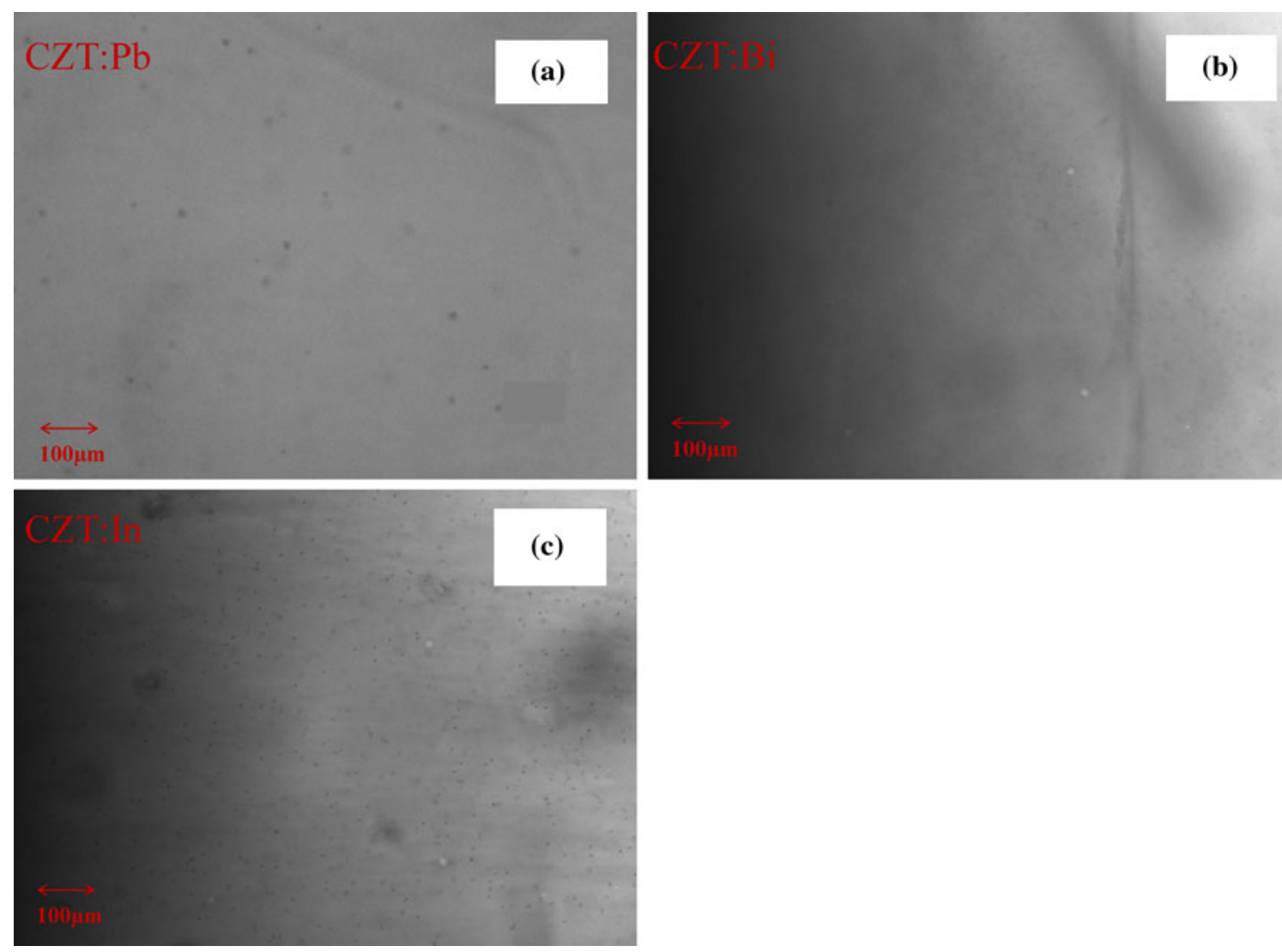

Fig. 2. IR transmission images showing different Te secondary phases (dark spots) for three detectors: (a) CZT:Pb, medium and small phases; (b) CZT:Bi, small phases, almost negligible concentration; and (c) CZT:In, high concentration of small phases.

likelihood of $\mathrm{Pb}$ substitutions on $\mathrm{Cd}$ vacancies, forming a donor level $\left[\mathrm{Pb}_{\mathrm{Cd}}\right]^{+}$; second, there is the chance that the new donor trap $\left[\mathrm{Pb}_{\mathrm{Cd}}\right]^{+}$might result in a deep acceptor level with $\mathrm{V}_{\mathrm{Cd}}$, in the form of $\left[\left[\mathrm{Pb}_{\mathrm{Cd}}\right]^{+}-\mathrm{V}_{\mathrm{Cd}}^{2-}\right]^{-}$If the 1.1-eV trap mentioned earlier is a donor level trap, then to attain high resistivity in CZT:Pb, the Pb-related trap should be an acceptor. To discover the nature of these traps, we carried out some TEES measurements, as shown in Fig. $1 b$. Two acceptor levels were identified. Hence, our assumption that the complex $\left[\left[\mathrm{Pb}_{\mathrm{Cd}}\right]^{+}-\mathrm{V}_{\mathrm{Cd}}^{2-}\right]^{-}$is formed is the more acceptable alternative. Savitsky et al. ${ }^{10}$ also identified a Pb-related acceptor level at about $E_{\mathrm{v}}+(0.38-0.43) \mathrm{meV}$. The $1-\mathrm{eV}$ to $1.2-\mathrm{eV}$ traps that we found earlier in almost all the CZT ${ }^{4}$ and cadmium manganese telluride $(\mathrm{CMT})^{11}$ detectors had remarkably high levels of Te secondary phases; hence, we conjecture that the trap around $1 \mathrm{eV}$ to $1.2 \mathrm{eV}$ might reflect the presence of Te precipitates. IR transmission measurements verified the presence of Te secondary phases in these three detectors; Fig. 2 displays the images. The IR images for CZT:Pb and CZT:In show large and small Te phases (Fig. 2a and c, respectively). On the other hand, Bi-doped crystals (Fig. 2b) had negligible numbers of Te phases, as seen by IR microscopy. In addition, as discussed later, the Bi-doped detectors lacked the 1.1-eV trap (Fig. 3a); hence, our assumption relating the $1.1-\mathrm{eV}$ trap in CZT detectors with Te precipitates seems likely.

\section{$C Z T: B i$}

Two types of Bi-doped CZT detectors, denoted CZT:Bi-B and CZT:Bi-Bro, with dopant concentration of $\sim 10^{19} \mathrm{~cm}^{-3}$, were characterized for point defects. The difference between them was in the $\mathrm{Zn}$ concentration and the Cd overpressure used during crystal growth. The $\mathrm{Zn}$ concentration for CZT:Bi-B and CZT:Bi-Bro, respectively, was $7.5 \%$ and $15 \%$. In addition, the latter was grown in Cd overpressure, while the former was grown under normal conditions. Figure 3 shows I-DLTS plots with energy levels for both detectors; they show two distinct dominant traps: a shallow one around $0.03 \mathrm{eV}$ and a deep trap of $0.82 \mathrm{eV}$ in CZT:Bi-B and CZT:Bi-Bro, respectively. Saucedo et al. ${ }^{12}$ reported a deep trap in the same energy range in CdTe:Bi-doped detectors. Hence, this difference is unrelated to the $\mathrm{Zn}$ content, leaving only the difference in Cd overpressure during crystal growth. The plots reveal that the $\mathrm{Cd}$ overpressure enhances the $0.82-\mathrm{eV}$ trap and decreases the $36-\mathrm{meV}$ trap. Elemental $\mathrm{Bi}$ has a binary action, and can occupy Cd- or Te-lattice positions. Accordingly, relating this with the presence of $\mathrm{V}_{\mathrm{Cd}}$ in a non-Cd atmosphere, the dominance 

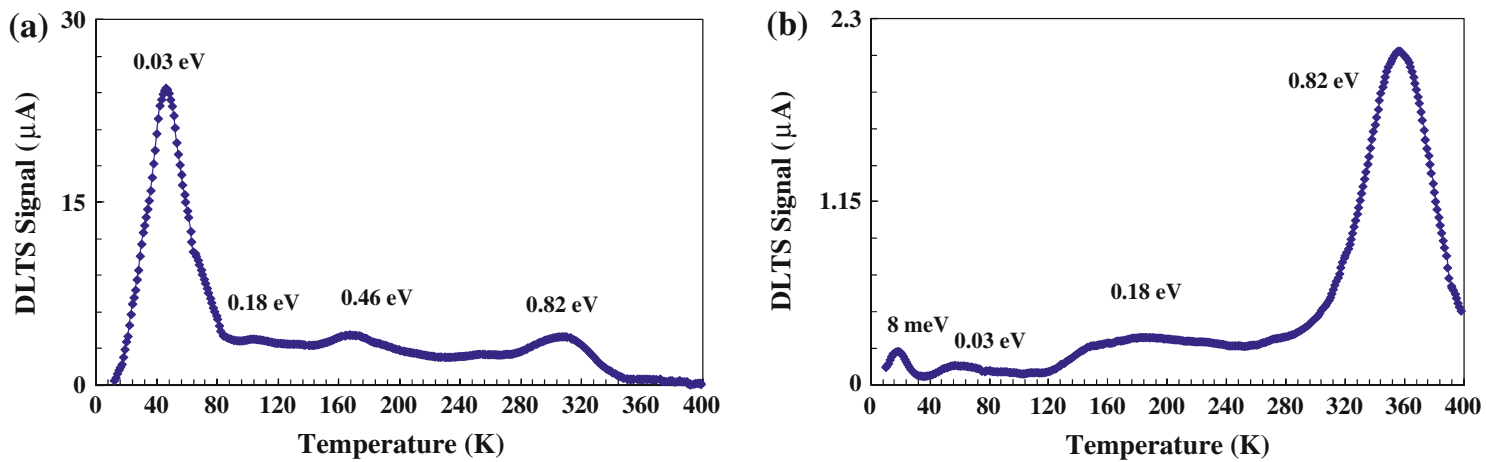

Fig. 3. DLTS spectrum with defects: (a) CZT:Bi-B, the shallow trap at $0.03 \mathrm{eV}$ is dominant; (b) CZT:Bi-Bro, the dominant deep trap at $0.82 \mathrm{eV}$ causes an increase in the resistivity of the material.

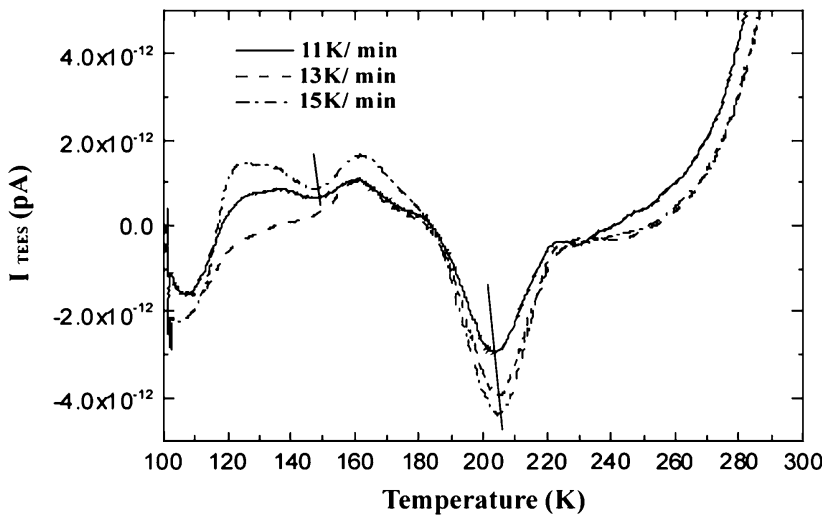

Fig. 4. TEES measurements for CZT:Bi-Bro with a deep donor trap at around $210 \mathrm{~K}$.

of the $36-\mathrm{meV}$ trap can be attributed to $\mathrm{Bi}_{\mathrm{Cd}}$. In this way, Bi compensates almost all $\mathrm{Cd}$ vacancies for this level of Bi doping. Another piece of evidence for this conclusion is that, similar to the Pd-doped detector, the trap around $0.31 \mathrm{eV}$ attributed to $\mathrm{V}_{\mathrm{Cd}}$ was not identified in these Bi-doped detectors. In $\mathrm{CZT}$ :Bi-Bro, the $\mathrm{Cd}$ overpressure resulted in the dominance of the $0.82-\mathrm{eV}$ trap. The donor nature of the trap is evident from the TEES plot in Fig. 4. The deep nature of the trap indicates that it does not just represent the compensation of intrinsic defects, but most probably reflects the formation of some complex center. Park and Chadi, ${ }^{13}$ in their firstprinciples pseudopotential calculations, identified a low-energy lattice instability state (VI-VI dimer bonding, Te-Te in our case) that is most effective in acceptor passivation $\left(\mathrm{V}_{\mathrm{Cd}}\right.$ in our case) in II-VI semiconductors. Formation of a deep donor trap is reported as being the result of these structural changes in a crystal. ${ }^{12,13}$

Figure 5 shows the dark current and the response to 822-nm light for CZT:Bi-B. The calculated values of resistivities for CZT:Bi-B and CZT:Bi-Bro, respectively, are $10^{8} \Omega \mathrm{cm}$ and $10^{9} \Omega \mathrm{cm}$. The deep traps close to the Fermi level mostly are responsible for the material's high resistivity. The presence of the $0.82-\mathrm{eV}$ trap in the latter, i.e., the comparatively

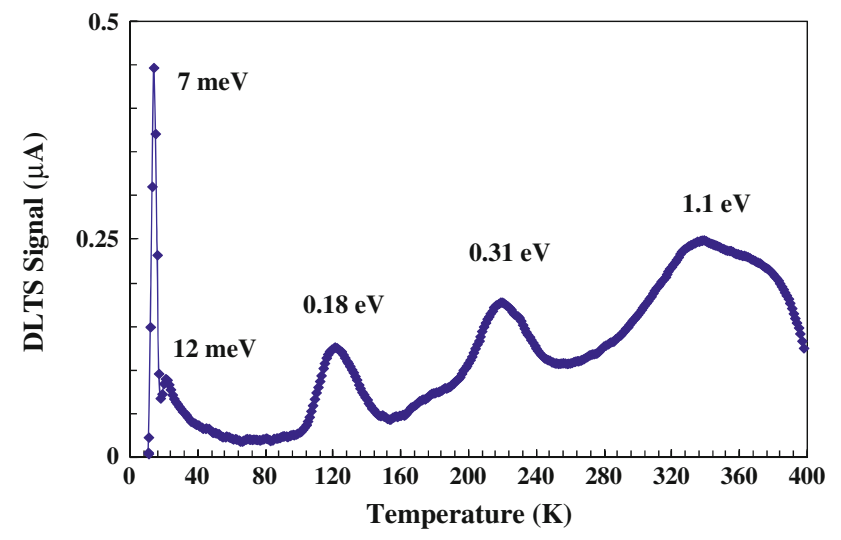

Fig. 5. CZT:In DLTS spectrum with defects labeled for $\lambda=822 \mathrm{~nm}$.

higher resistivity detector of the two, supports this statement. Saucedo et al. ${ }^{12}$ also related the deep trap they found in Bi-doped CdTe detectors with the material's high resistivity and high photoconductivity.

Besides the Bi-related traps, we identified a few more traps, one of them with a weak DLTS signal at around $0.46 \mathrm{eV}$. As seen from the glow-discharge mass spectrometry (GDMS) data in Table II, Fe is found in high concentrations. Based on the findings from our new Fe-doped detector (soon to be reported) and the GDMS data, we consider that the $0.46-\mathrm{eV}$ trap is related to Fe impurities.

An A-center ${ }^{4}$ with a weak DLTS signal can also be seen at around $0.18 \mathrm{eV}$, as shown in Fig. 3. The origin of this A-center is the formation of a complex between $\left[\mathrm{V}_{\mathrm{Cd}}\right]^{2-}$ and $\left[\mathrm{M}_{\mathrm{Cd}}\right]^{+}$, where here $\mathrm{M}$ corresponds to In $\left(5.7 \times 10^{15}\right.$ at. $\left.\mathrm{cm}^{-3}\right)$ or $\mathrm{Al}$ $\left(5 \times 10^{15}\right.$ at. $\left.\mathrm{cm}^{-3}\right)$ impurities in CZT:Bi. Compared with other impurities, In and $\mathrm{Al}$ have a high chance of occupying the $\mathrm{V}_{\mathrm{Cd}}$ position. The other shallow traps due to elemental metallic impurities also are evident.

The 1.1-eV trap corresponding to Te precipitates was absent in Bi-doped detectors, as also verified by the IR image for this detector (Fig. 2b), wherein there are almost no Te-rich phases, thus confirming 
our statement relating the $1.1-\mathrm{eV}$ trap with $\mathrm{Te}$ precipitates.

\section{CZT:In}

Figure 5 plots the DLTS findings, showing the energy traps in the CZT:In detector. Two shallow traps at around $7 \mathrm{meV}$ and $12 \mathrm{meV}$ were identified, which we again attributed to the metallic elemental impurities. There are two additional well-known acceptor levels, A-centers, at around $0.18 \mathrm{eV}$, and $\left[\mathrm{V}_{\mathrm{Cd}}\right]^{2-}$ at around $0.31 \mathrm{eV}$. The presence of these two traps indicates that In has partially compen-

Table 2. GDMS data for CZT:Bi-B detector

\begin{tabular}{lc}
\hline Impurity & $\begin{array}{c}\text { Concentration } \\
\left(\text { at. }^{-3} \text { ) }\right.\end{array}$ \\
\cline { 2 - 2 } $\mathrm{Li}$ & $5.2 \times 10^{16}$ \\
$\mathrm{Na}$ & $1.0 \times 10^{16}$ \\
$\mathrm{Mg}$ & $5.4 \times 10^{15}$ \\
$\mathrm{Al}$ & $5.0 \times 10^{15}$ \\
$\mathrm{~K}$ & $2.0 \times 10^{15}$ \\
$\mathrm{Ca}$ & $3.6 \times 10^{15}$ \\
$\mathrm{Cu}$ & $1.0 \times 10^{16}$ \\
$\mathrm{Ag}$ & $1.2 \times 10^{15}$ \\
$\mathrm{In}$ & $5.7 \times 10^{15}$ \\
$\mathrm{Ga}$ & $1.4 \times 10^{16}$ \\
$\mathrm{Bi}$ & $2.4 \times 10^{19}$ \\
\hline
\end{tabular}

sated the Cd vacancies, by making an A-center. The 1.1-eV trap identified in $\mathrm{CZT}: \mathrm{Pb}$ also was present in this detector.

\section{Capture Cross-Sections}

Figure 6 plots the capture cross-sections for the energy traps for the four detectors. It reveals that deep traps have a higher probability of capturing charge carriers. The values vary from $10^{-21} \mathrm{~cm}^{2}$ to $10^{-7} \mathrm{~cm}^{2}$, from shallow traps to deep traps, respectively.

\section{Electrical and Radiation Responses}

The electrical response of the detectors was assessed by $I-V$ experiments. The dark current measured for the Bi-doped detectors was comparatively higher than for the other two. The resistivities recorded in Table I were calculated from the $I-V$ measurements. The $\mathrm{Pb}$ - and In-doped detectors exhibited high resistivity of $\sim 10^{10} \Omega \mathrm{cm}$. The Bi dopant conferred high resistivity in CdZnTe, but the detector failed to show any response to incident gamma radiation. In- and $\mathrm{Pb}$-doped detectors showed radiation response. The mobility-lifetime product of these detectors was $0.7 \times 10^{-3} \mathrm{~cm}^{2} \mathrm{~V}^{-1}$ to $2 \times 10^{-3} \mathrm{~cm}^{2} \mathrm{~V}^{-1}$. Photoresponse to white light and 822-nm light-emitting diode (LED) light was observed for all three detectors. The response of the Bi-doped detector is shown as an example in Fig. 7.
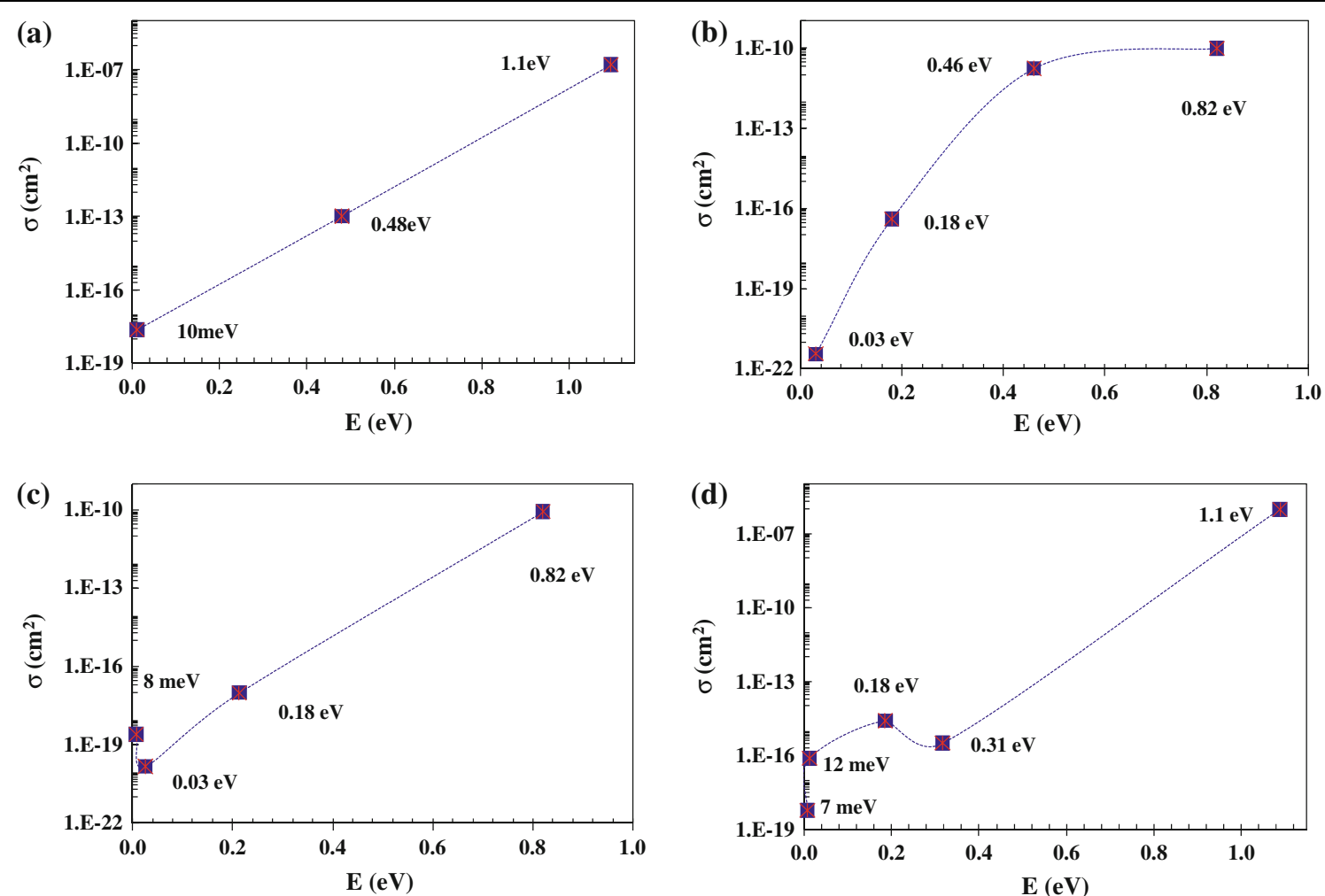

Fig. 6. Capture cross-sections for four detectors: (a) CZT:Pb, (b) CZT:Bi-B, (c) CZT:Bi-Bro, and (d) CZT:In. The values vary from $10^{-21} \mathrm{~cm}^{2}$ to $10^{-7} \mathrm{~cm}^{2}$, for shallow to deep traps, respectively. 


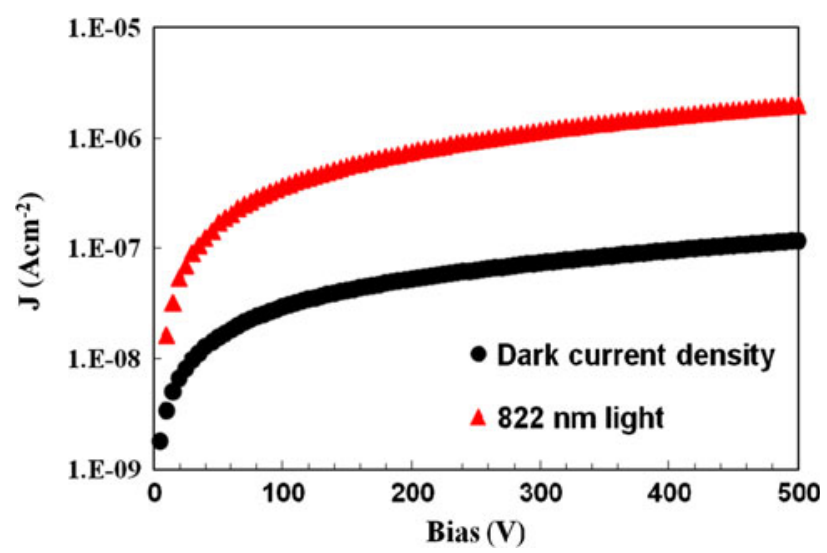

Fig. 7. Dark current density and photoresponse of CZT:Bi-B detector to 822-nm light.

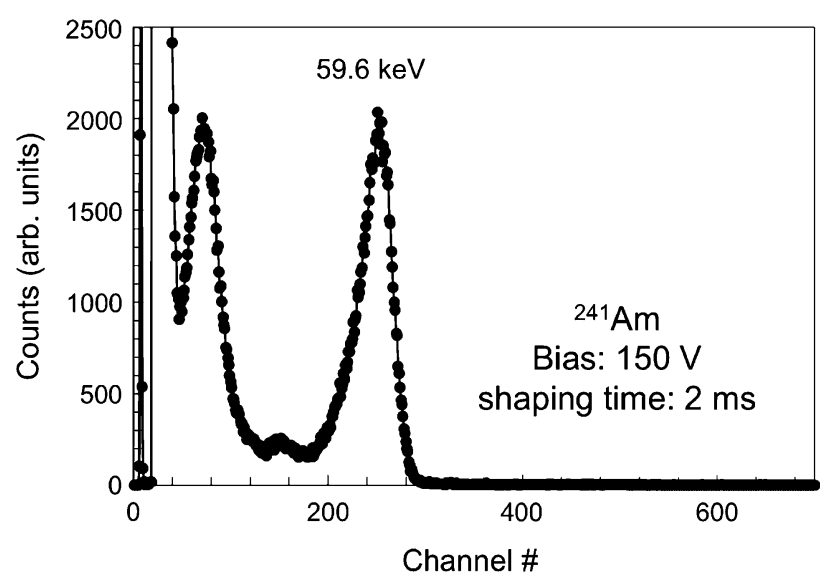

Fig. 8. CZT:In detector response to gamma rays; the full-width at half-maximum (FWHM) of the $59.6-\mathrm{keV}$ peak is $11.2 \%$.

$\mathrm{Pb}$ - and $\mathrm{Bi}$-doped detectors showed no response to gamma rays, while the 2 -mm-thick In-doped detectors responded to them (Fig. 8). In case of $\mathrm{Pb}$-doped CZT detectors, a 9-mm-thick detector had a good response; the results have been published elsewhere. $^{14}$

\section{CONCLUSIONS}

We investigated $\mathrm{CdZnTe}$ detectors doped with $\mathrm{Pb}$, $\mathrm{Bi}$, and In. We identified distinct traps in each, most probably related to the particular dopant in the crystal. In $\mathrm{Pb}$ - and $\mathrm{Bi}$-doped CZT detectors, almost all the $\mathrm{V}_{\mathrm{Cd}}$ are compensated by $\mathrm{Pb}$ and $\mathrm{Bi}$ atoms. In the former, an expected new deep acceptor complex, with energy of $0.48 \mathrm{eV}$, is induced within the bandgap. Bi-doped CZT crystals have a shallow and a deep donor at around $0.03 \mathrm{eV}$ and $0.82 \mathrm{eV}$; the latter strongly influences the resistivity of the detectors. In-doped CZT crystals have well-known A-centers and dominant Cd-vacancy-related acceptors at around $0.18 \mathrm{eV}$ and $0.31 \mathrm{eV}$. Based on the DLTS measurements and the electrical and photoresponses, we conclude that, in CdZnTe, the In dopant is comparatively more applicable for roomtemperature radiation detectors.

\section{ACKNOWLEDGEMENTS}

This work was supported by the Defense Treat Reduction Agency and US Department of Energy, Office of Nonproliferation Research and Development, NA-22.

\section{REFERENCES}

1. C. Szeles, Phys. Stat. Sol. (b) 241, 783 (2004).

2. R.B. James, T.E. Schlesinger, J. Lund, and M. Schieber, Semiconductors and Semimetals, Vol. 43 (New York: Academic, 1997), p. 335.

3. M. Schieber, T.E. Schlesinger, R.B. James, H. Hermon, H. Yoon, and M. Goorsky, J. Cryst. Growth 2082, 237 (2002).

4. R. Gul, A.E. Bolotnikov, K.H. Kim, R. Rodriguez, K. Keeter, Z. Li, G. Gu, and R.B. James, J. Electron. Mater. 40, 274 (2011).

5. Z. Li, Nucl. Instrum. Meth. A 403, 399 (1998).

6. A. Zappettini, M. Zha, M. Pavesi, and L. Zanotti, J. Cryst. Growth 307, 283 (2007).

7. E. Saucedo, L. Fornaro, N.V. Sochinskii, A. Cuña, V. Corregidor, D. Granados, and E. Diéguez, IEEE Trans. Nucl. Sci. 51, 3105 (2004).

8. H. Elhadid, J. Franc, P. Moravec, P. Hoschl, and M. Fiederle, Semicond. Sci. Technol. 22, 537 (2007).

9. A.E. Bolotnikov, G.S. Camarda, G.A. Carini, Y. Cui, K.T. Kohman, L. Li, M.B. Salomon, and R.B. James, IEEE Trans. Nucl. Sci. 54, 821 (2007).

10. A.V. Savitskya, M.I. Ilashchuka, O.A. Parfenyuka, K.S Ulyanytskya, V.R. Buracheka, R. Ciach, Z. Swiatekb, and Z. Kuznickic, Thin Solid Films 361-362, 203 (2000).

11. R. Gul, K.H. Kim, A.E. Bolotnikov, G.S. Carmarda, G. Yang, A. Hossain, Y. Cui, and R.B. James (unpublished).

12. E. Saucedo, C.M. Ruiz, V. Bermúdez, E. Dieguez, E. Gombia, A. Zappettini, A. Baraldi, and N.V. Sochinskii, J. Appl. Phys. 100, 104901 (2006).

13. C.H. Park and D.J. Chadi, Phys. Rev. Lett. 75, 1135 (1995).

14. K.H. Kim, R. Gul, V. Carcelen, A.E. Bolotinkov, G.S. Carmarda, G. Yang, A. Hossain, Y. Cui, R.B. James, J. Hong, and S.U. Kim, J. Cryst. Growth 312, 781 (2010). 